\title{
The mechanism of the additive action of bacterial compositions Bacillus spp. in the defense response of common wheat against greenbug aphid Schizaphis graminum
}

\author{
Rumyantsev S.D., Alekseev V.Y., Veselova S.V., Cherepanova E.A., \\ Burkhanova G.F., Maksimov I.V. \\ Institute of Biochemistry and Genetics of the UFRC of the RAS, Ufa, Russia \\ * email: rumyantsev-serg@mail.ru
}

Biocontrol agents based on plant-growth promoting bacteria (PGPB) can become an effective and environmentally safe means of plant protection agents against pests. It is possible to enhance the effect of biocontrol agent by compiling bacterial compositions. However, in the world scientific literature, there is practically no information about the effect of the combined action of endophytic bacterial strains on the plant defense systems against pests. Bacterial metabolites are the active origin of any biocontrol product. Our work was aimed at studying the molecular and biochemical mechanisms of action of various combinations of bacterial metabolites of Bacillus spp. on the defense system of wheat plants during the colonization of the greenbug aphid Schizaphis graminum. In our work, we studied 3 bacterial strains that synthesize different groups of metabolites. Thus, the strains of B. subtilis 26D and B. subtilis 11BM produce lipopeptides - surfactin and iturin respectively. The $B$. thuringiensis strain B-5351 synthesizes Cry proteins. The insecticidal activity of the mixture $B$. thuringiensis B-5351+B. subtilis 26D was higher than that of individual strains. All three bacterial mixtures $B$. thuringiensis $\mathrm{B}-5351+B$. subtilis 26D, B. subtilis 26D+B. subtilis 11BM, B. thuringiensis B-5351+B. subtilis $11 \mathrm{BM}$ increased the growth-promoting effect compared to the action of individual strains. All strains and their compositions had an indirect effect on aphid mortality and the formation of induced systemic resistance of wheat plants against $S$. graminum, but the mixture $B$. thuringiensis $\mathrm{B}-5351+B$. subtilis $26 \mathrm{D}$ had the greatest effect. When plants were treated with this mixture, aphid propagation was the lowest, and the accumulation of hydrogen peroxide and the induction of $P R$-genes (from pathogenesis related) expression were the highest.

Acknowledgements: This work was supported by the RFBR in the framework of the research project No. 20-316-90021. 